\title{
Swimming exercise ameliorates mood disorder and memory impairment by enhancing neurogenesis, serotonin expression, and inhibiting apoptosis in social isolation rats during adolescence
}

\author{
Hye-Sang Park', Tae-Woon Kim²,3, Sang-Seo Park'2, Sam-Jun Lee ${ }^{4, *}$ \\ 'Department of Kinesiology, College of Public Health and Cardiovascular Research Center, Lewis Katz school of Medicine, Temple University, Philadelphia, PA, USA \\ 2Department of Physiology, College of Medicine, Kyung Hee University, Seoul, Korea \\ ${ }^{3}$ Exercise Rehabilitation Research Institute, Department of Exercise \& Health Science, Sangmyung University, Seoul, Korea \\ ${ }^{4}$ Department of Sport Rehabiliation, Tongmyong University, Busan, Korea
}

Social isolation during adolescence is associated with anxiety, depression, and memory impairment. Exercise has been reported as a positive effect on brain function, especially hippocampus. The present study examined the effect of swimming exercise on apoptosis, cell proliferation, and serotonin expression in social isolation rats during adolescence stage. Social isolation started at postnatal day 21 and continued for 6 weeks. The rats in the swimming group were forced to swim for $60 \mathrm{~min}$ once daily during 6 days per week for 6 consecutive weeks. The rats in the social isolation during adolescence showed anxiety, depression, short-term memory impairment. Social isolation facilitated apoptosis and inhibited cell proliferation and differentiation. Social isolation sup- pressed expression of serotonin, brain-derived neurotrophic factor, and tyrosine kinase B. Swimming exercise alleviated anxiety, depression, short-term impairment. Swimming exercise suppressed apoptosis, enhanced neurogenesis, and increased serotonin expression. In our study, swimming exercise ameliorates mood disorder and memory impairment by enhancing neurogenesis and serotonin expression and inhibiting apoptosis in social isolation.

Keywords: Social isolation, Adolescence, Anxiety, Depression, Short-term memory, Swimming

\section{INTRODUCTION}

Social isolation during adolescence causes depressive symptoms and reduces memory function (Dunphy-Doherty et al., 2018). Social isolation at a young age inhibits dendritic development in the hippocampus and induces neurological dysfunction in the limbic system (Silva-Gómez et al., 2003). The hippocampus is an important region of the brain involved in the process of learning and memory formation, and hippocampal neurogenesis continues to take place after the development of the nervous system (López-Toledano and Shelanski, 2004).
Apoptosis is a form of cell death that serves to remove dying cells from cell proliferating or differentiating, thus apoptosis plays a critical role in normal development and tissue homeostasis. Inappropriate or excessive apoptosis, however, is implicated in diverse neurological disorders (Lee et al., 2003). Terminal deoxynucleotidyl transferase-mediated dUTP nick end labeling (TUNEL) assay detects DNA fragmentation, one of the character-istics of apoptotic cell death (Gavrieli et al., 1992). Bcl-2 inhibits apoptosis while Bax promotes apoptosis (Song et al., 2018).

Brain-derived neurotrophic factor (BDNF) and its receptor, tyrosine kinase $\mathrm{B}(\mathrm{TrkB})$, are abundantly expressed in the mamma-
${ }^{*}$ Corresponding author: Sam-Jun Lee (iD https://orcid.org/0000-0002-8491-3602 Department of Physical Education, College of Health, Social Welfare, and Education, Tongmyong University, 428 Sinseonno, Nam-gu, Busan 48520, Korea E-mail: anada23@tu.ac.kr

Received: February 15, 2020 / Accepted: March 27, 2020
This is an Open Access article distributed under the terms of the Creative Commons Attribution Non-Commercial License (https://creativecommons.org/licenses/by-nc/4.0/) which permits unrestricted non-commercial use, distribution, and reproduction in any medium, provided the original work is properly cited. 
lian brain, especially hippocampus. Learning and exercise increase hippocampal BDNF expression (Cho et al., 2017; Hall et al., 2000). Exercise-induced BDNF expression facilitates neurogenesis and enhances long-term potentiation of the hippocampus (Farmer et al., 2004).

Immunohistochemistry detects 5-bromo-2'-deoxyuridine (BrdU), and BrdU-positive cells represent newly formed cells (Park et al., 2019). Doublecortin (DCX) is a marker of neuronal precursor cells, and DCX is implicated in neuronal differentiation (Park et al., 2019).

Serotonin (5-hydroxytryptamine, 5-HT) is related with depression or anxiety (Wang et al., 2019). Tryptophan hydroxylase (TPH) is a speed-limiting enzyme for the production of 5-HT and TPH level is an indicator of 5-HT synthesis. Stress from social isolation leads to depression- and anxiety-related behaviors as well as neuroendocrine and physiologic dysfunctions that affect dopamine and 5-HT (McNeal et al., 2018).

Previous studies demonstrated that physical exercise affects therapeutic effects on anxiety, depression, and stress (Ji et al., 2017; Park et al., 2017). The present study examined the effect of swimming exercise on apoptosis, cell proliferation, and serotonin expression in the adolescence rats.

\section{MATERIALS AND METHODS}

\section{Experiment animals}

This study was approved by the Kyung Hee University Institutional Animal Care and Use Committee (Seoul, Korea) (KHUASP [SE]-16-154). Male Wistar rats were randomly divided into 4 groups ( $n=8$ per group): group housed (GH), group housed and exercise $(\mathrm{GH}+\mathrm{E})$, socially isolated (SI), and socially isolated and exercise (SI+E). Social isolation was performed as described by Amiri et al. (2015). At postnatal day 21, the rats were randomly housed for 6 weeks under two different conditions: GH and SI. GH rats were housed (4 per cage) in Plexiglas boxes and SI rats were housed individually in Plexiglas boxes.

\section{Swimming exercise protocol}

The rats in the swimming group were forced to swim for $60 \mathrm{~min}$ once daily during 6 days per week for 6 consecutive weeks. In swimming pool $(120 \mathrm{~cm} \times 80 \mathrm{~cm} \times 50 \mathrm{~cm})$, filled with water at a temperature of $30^{\circ} \mathrm{C} \pm 2{ }^{\circ} \mathrm{C}$. Swimming was performed weighing $5 \%$ of the rat body weight.

\section{Elevated plus-maze test}

Anxiety-like behavior was evaluated using the elevated plus-maze test as previous method (Park et al., 2019). The elevated plus-maze apparatus consisted of two open arms $(45 \mathrm{~cm} \times 10 \mathrm{~cm})$, crossed at right two opposed arms of the same size. The junction area of central measured platform $(10 \mathrm{~cm} \times 10 \mathrm{~cm})$, set up $65 \mathrm{~cm}$ above the floor. The rats were placed on the central platform facing a closed arm and were allowed to explore the maze freely for $5 \mathrm{~min}$. Entry into an arm was defined as entry of all four paws into the arm. Times and entries in the open arms were measured.

\section{Forced swimming test}

The forced swimming test was used to confirm a depressive state as previous method (Lee et al., 2019). The experimental animals were placed individually in glass cylinders (height, $50 \mathrm{~cm}$; diameter, $15 \mathrm{~cm}$ ) containing water at a temperature of $25^{\circ} \mathrm{C}$ and a depth of approximately $30 \mathrm{~cm}$. All rats underwent a $15-\mathrm{min}$ pretest to confirm their adaptation to water. One day after the pretest, the animals were tested for $5 \mathrm{~min}$. During the test session, immobility time was measured using the smart version 2.5 video tracking system. Immobility was defined to occur when no additional activity was observed other than the actions needed by the animal to keep its head above the water.

\section{Step-through avoidance test}

In order to evaluate short-term term memory, step-through avoidance test was performed at $24 \mathrm{hr}$ after training as previous method (Park et al., 2019). The latency time (sec) in each group was measured. In the training session, the rat was placed in illuminated compartment, and then guillotine door was raised to allow the rat to enter the dark compartment. When the rat's hind leg entered the dark compartment, the guillotine door was closed. Electrical foot shock $(0.2 \mathrm{~mA})$ was delivered through the grid floor for $2 \mathrm{sec}$. At $24 \mathrm{hr}$ after training session, this process performed again. The time that elapsed prior to entry into dark compartment was recorded. A latency time over $180 \mathrm{sec}$ was counted as $180 \mathrm{sec}$.

\section{Tissue preparation}

The animals were sacrificed immediately after determination of step-through avoidance test. To prepare the brain slices, the animals were fully anesthetized with ethyl ether, and then the rats were transcardially perfused with $50 \mathrm{mM}$ phosphate-buffered saline. The rats were fixed with freshly prepared solution of $4 \%$ paraformaldehyde in $100 \mathrm{mM}$ phosphate buffer $(\mathrm{pH}, 7.4)$. The brains were then removed, postfixed in the same fixative overnight, and 
transferred into a $30 \%$ sucrose solution for cryoprotection. Coronal sections with thickness of $40 \mu \mathrm{m}$ were made using a freezing microtome (Leica, Nussloch, Germany).

\section{Immunohistochemistry for TPH, 5-HT in dorsal raphe}

To visualize TPH and 5-HT expression, immunohistochemistry for TPH and 5-HT in the dorsal raphe nuclei was performed as previously described method (Park et al., 2019). The sections were incubated in phosphate-buffered saline (PBS) for $10 \mathrm{~min}$, and then washed 3 times in the same buffer. The sections were then incubated in $1 \%$ hydrogen peroxide for $30 \mathrm{~min}$. The sections were incubated overnight with rabbit anti-TPH antibody (1:1,000; Oncogene Research Product, Cambridge, UK), rabbit anti-5-HT antibody (1:500; Abcam, Cambridge, UK) and goat anti-DCX antibody (1:1,000; Oncogene Research Product) and then with biotinylated rabbit and goat secondary antibody (1:200; Vector Laboratories, Burlingame, CA, USA) for another $1 \mathrm{hr}$. The secondary antibody was amplified with the Vector Elite ABC kit (1:100; Vector Laboratories). Antibody-biotin-avidin-peroxidase complexes were visualized using $0.03 \%$ diaminobenzidine (DAB), and the sections were mounted onto gelatin-coated slides. The slides were air-dried overnight at room temperature, and the coverslips were mounted using Permount (Thermo Fisher Scientific Inc., Waltham, MA, USA).

\section{Immunohistochemistry for BrdU}

Immunohistochemistry for $\mathrm{BrdU}$ was performed as previously described method (Park et al., 2019). BrdU (50 mg/kg; Sigma Chemical Co., St. Louis, MO, USA) was given intraperitoneally to all animals $1 \mathrm{hr}$ before starting treadmill exercise for the 3 consecutive days prior to sacrifice. The sections were first permeabilized by incubation in $0.5 \%$ Triton X-100 in PBS for $20 \mathrm{~min}$, then pretreated in $50 \%$ formamide- $2 \mathrm{X}$ standard saline citrate at $65^{\circ} \mathrm{C}$ for $2 \mathrm{hr}$, denatured in $2 \mathrm{~N} \mathrm{HCl}$ at $37^{\circ} \mathrm{C}$ for $30 \mathrm{~min}$, and rinsed twice in $100 \mathrm{mM}$ sodium borate $(\mathrm{pH}, 8.5)$. The sections were then incubated overnight at $4{ }^{\circ} \mathrm{C}$ with $\mathrm{BrdU}$-specific mouse monoclonal antibody (1:600; Roche, Mannheim, Germany). They were then washed three times with PBS and incubated with biotinylated mouse secondary antibody (1:200; Vector Laboratories) for $1 \mathrm{hr}$. The sections were then incubated for $1 \mathrm{hr}$ with an $\mathrm{ABC}$ complex (1:100; Vector Laboratories). For visualization, sections were incubated in $50 \mathrm{mM}$ Tris- $\mathrm{HCl}(\mathrm{pH}, 7.6)$ containing $0.03 \% \mathrm{DAB}$, $40 \mathrm{mg} / \mathrm{mL}$ nickel chloride, and $0.03 \%$ hydrogen peroxide for $5 \mathrm{~min}$. After BrdU labeling, a mouse antineuronal nucleic antibody (1: 1,000; Chemicon International, Temecula, CA, USA) was used on the same sections to differentiate neurons. The sections were mounted onto gelatin-coated slides, air-dried overnight at room temperature, and the coverslips were mounted using Permount (Thermo Fisher Scientific Inc.).

\section{Immunohistochemistry for DCX}

Immunohistochemistry for DCX was performed as previously described method (Park et al., 2019). The sections were incubated in PBS for 10 min, washed three times in PBS, and then incubated in $1 \%$ hydrogen peroxide for $20 \mathrm{~min}$. The sections were incubated $2 \mathrm{hr}$ with goat anti-DCX antibody (1:1,000; Oncogene Research Product). The sections were then incubated with the biotinylated goat secondary antibody (1:500; Vector Laboratories) for another $1 \mathrm{hr}$, washed, and incubated in $\mathrm{ABC}$ complex (Vector Elite $\mathrm{ABC}$ kit; 1:100; Vector Laboratories). Labeling was visualized using $0.03 \% \mathrm{DAB}$, and the sections were mounted onto gelatin-coated slides. The slides were air-dried overnight at room temperature, and the coverslips were mounted using Permount (Thermo Fisher Scientific Inc.)

\section{TUNEL staining}

To visualize DNA fragmentation, we performed TUNEL staining using an In Situ Cell Death Detection Kit (Roche) according to the manufacturer's protocol as previously described method (Song et al., 2018). The sections were postfixed in ethanol-acetic acid $(2: 1)$ and rinsed, then incubated with proteinase $\mathrm{K}(100 \mathrm{mg} / \mathrm{mL})$ and rinsed again. They were then incubated in $3 \%$ hydrogen peroxide, permeabilized with $0.5 \%$ Triton $\mathrm{X}-100$, rinsed again, and incubated in the TUNEL reaction mixture. The sections were rinsed and visualized using Converter-POD with $0.03 \% \mathrm{DAB}$, counterstained with Nissl and mounted onto gelatin-coated slides. The slides were air-dried overnight at room temperature and the coverslips were mounted using Permount (Thermo Fisher Scientific Inc.).

\section{Western blotting for BDNF, TrkB, Bax, and Bcl-2}

Western blotting for the determination of Bax, Bcl-2, BDNF, TrkB was conducted as previously described method (Park et al., 2019; Song et al., 2018). The hippocampus tissues were homogenized on ice and lysed in a lysis buffer containing $50 \mathrm{mM}$ Tris- $\mathrm{HCl}$ ( $\mathrm{pH}, 7.5), 150 \mathrm{mM} \mathrm{NaCl}, 0.5 \%$ deoxycholic acid, $1 \%$ Nonidet P40, $0.1 \%$ sodium dodecyl sulfate (SDS), $1 \mathrm{mM}$ phenylmethylsulfonyl fluoride, and $100-\mathrm{mg} / \mathrm{mL}$ leupeptin. Protein content was measured using a Bio-Rad colorimetric protein assay kit (Bio-Rad, Hercules, CA, USA). Protein of $30 \mu \mathrm{g}$ was separated on SDS-polyacrylamide gels and transferred onto a nitrocellulose membrane, 
which was incubated with mouse $\beta$-actin antibody (1:1,000; Santa Cruz Biotechnology, Santa Cruz, CA, USA), mouse Bax (1:1,000; Santa Cruz Biotechnology), Bcl-2 (1:1,000; Santa Cruz Biotechnology), and rabbit BDNF (1:1,000; Santa Cruz Bio-technology), TrkB (1:1,000; Santa Cruz Biotechnology). Horseradish peroxidase-conjugated anti-mouse for $\beta$-actin, Bax, Bcl-2 and anti-rabbit for BDNF, TrkB were used as secondary antibodies.

\section{Data analysis}

For confirming the expression of Bax, Bcl-2, BDNF, and TrkB, the detected bands were calculated densitometrically using Molecular Analyst version 1.4.1 (Bio-Rad). The numbers of 5-HTpositive and TPH-positive cells in the dorsal raphe and DCX-and BrdU-positive cells in the hippocampal dentate gyrus were counted hemilaterally under a light microscope (Olympus, Tokyo, Japan). The data were analyzed with one-way analysis of variance and then Duncan post hoc test. All values are expressed as the mean \pm stan- dard error of the mean (SEM), and $P$-value of $<0.05$ was considered significant.

\section{RESULTS}

\section{Effect of swimming exercise on anxiety, depression, and short-term memory}

Regarding anxiety in the elevated plus-maze test, the rats in the SI group showed anxiety compared to the $\mathrm{GH}$ group in open times and entries. Swimming exercise decreased anxiety level in the SI group (Fig. 1A, B). For depression in the forced swimming test, the rats in the SI group showed depression compared to the $\mathrm{GH}$ group in the immobility times. Swimming exercise alleviated depression level in the SI group (Fig. 1C). For short-term term memory in the step-through test, the rats in the SI group showed shortterm memory impairment compared to the GH group in latency times. Swimming exercise improved short-term memory in the SI
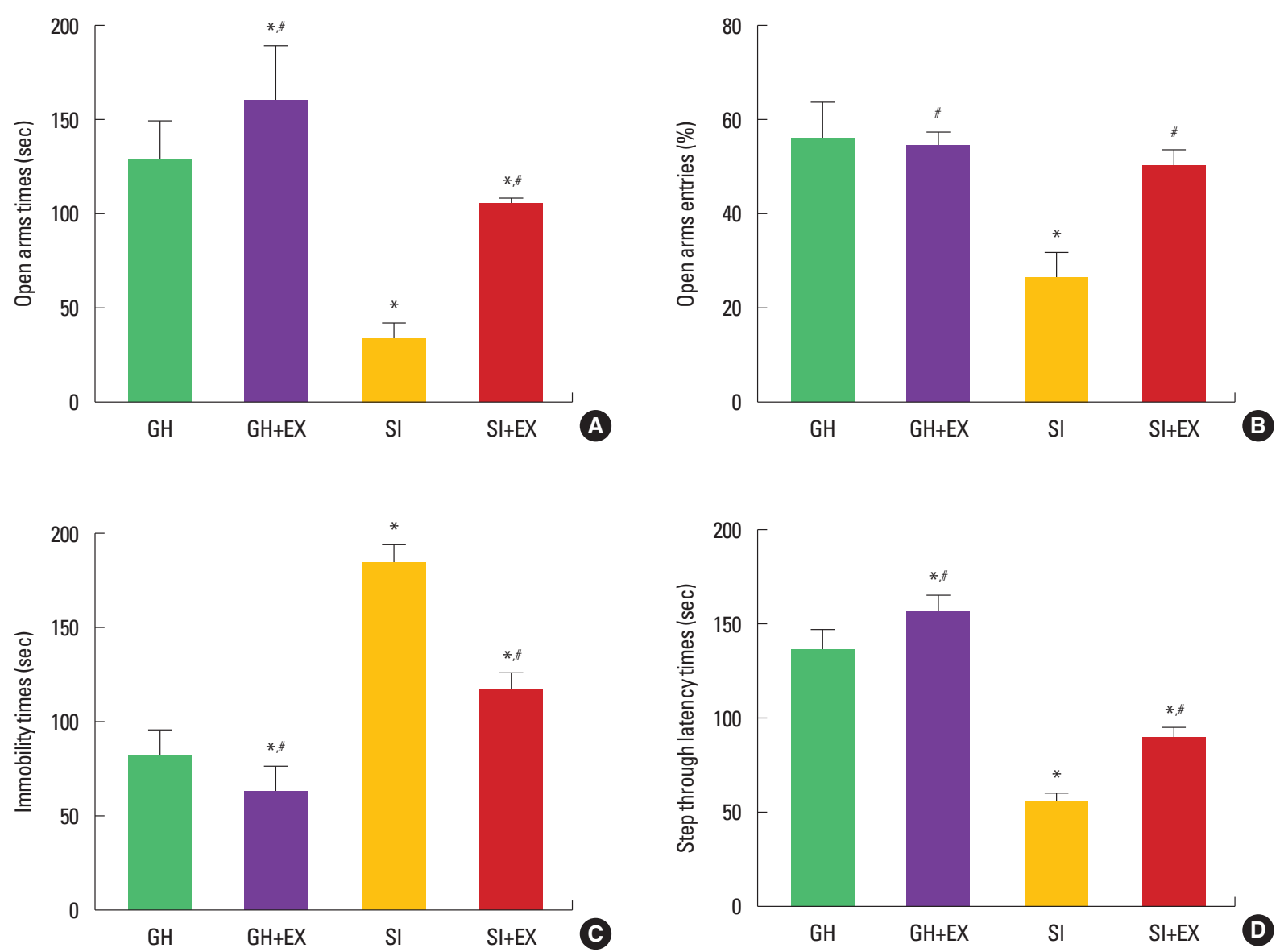

Fig. 1. Effect of swimming exercise on behaviors. Open arms times in elevated plus-maze test (A), open arms entries in elevated plus-maze test (B), immobility times in forced swimming test (C), latency times in step-through avoidance task (D). GH, group housed; GH+EX, group housed and exercise; SI, socially isolated; SI+EX, socially isolated and exercise. Data are expressed as the mean \pm standard error of the mean. ${ }^{*} P<0.05$ compared to the GH group. ${ }^{*} P<0.05$ compared to the $S \mathrm{~S}$ group. 
group (Fig. 1D).

\section{Effect of swimming exercise on apoptosis in the hippocampus}

When the expression of $\mathrm{Bax}$ and $\mathrm{Bcl}-2$ in $\mathrm{GH}$ group was set at 1.00 , the SI group showed an increase in Bax and a decrease in the $\mathrm{Bcl}-2$ compared to the GH group. Swimming exercise decreased Bax expression and increased $\mathrm{Bcl}-2$ expression in the SI group (Fig. 2A). The number of TUNEL-positive cells in the hippocampal dentate gyrus was increased in the SI group compared to the GH group. Swimming exercise decreased this number in the SI group (Fig. 2B).

\section{Effect of swimming exercise on BDNF and TrkB expression in the hippocampus}

When the expression of BDNF and $\operatorname{TrkB}$ in GH group was set
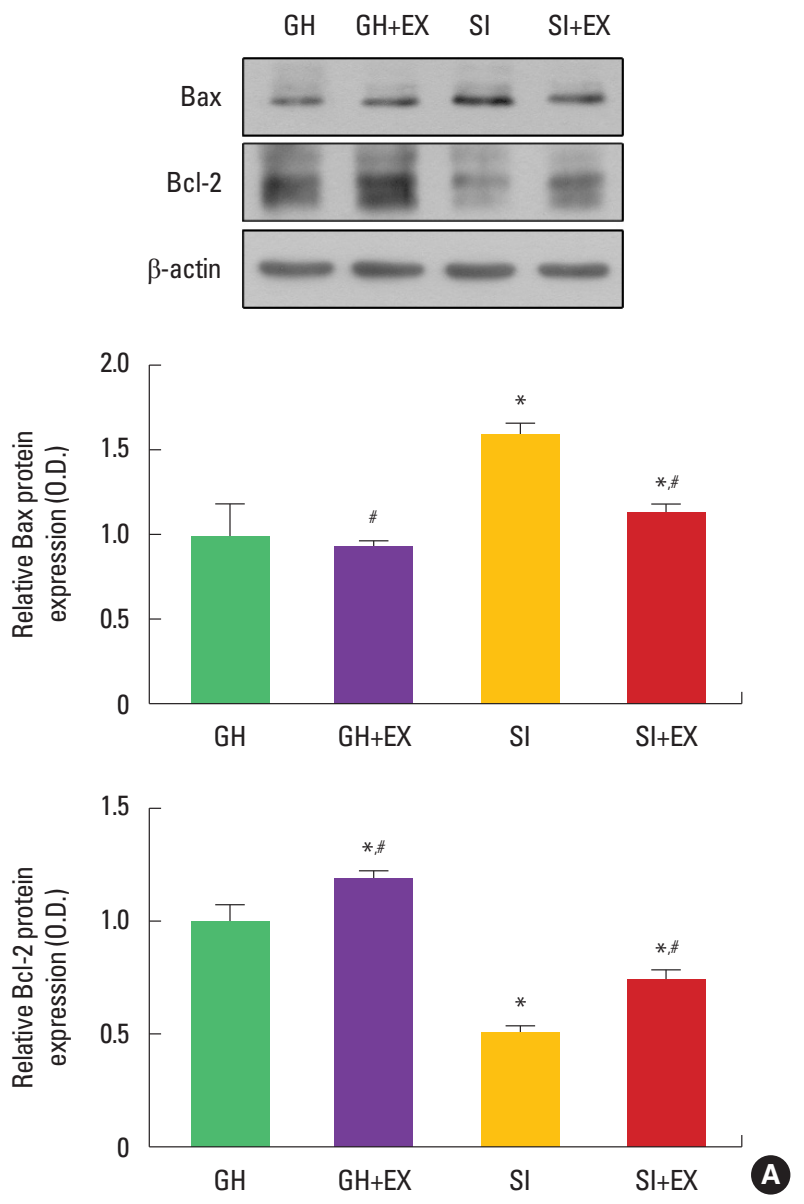

at 1.00, the SI group showed a decrease in BDNF and TrkB expression compared to the GH group. Swimming exercise increased BDNF and TrkB expression in the SI group (Fig. 3).

\section{Effect of swimming exercise on cell proliferation and differentiation in hippocampal dentate gyrus}

The number of BrdU-positive and DCX-positive cells in the hippocampal dentate gyrus was decreased in the SI group compared to the GH group. Swimming exercise increased these numbers in the SI group (Fig. 4).

\section{Effect of exercise on TPH and 5-HT in the dorsal raphe}

The number of TPH-positive and 5-HT-positive cells in the dorsal raphe was decreased in the SI group compared to the $\mathrm{GH}$ group. Swimming exercise increased these numbers in the SI group (Fig. 5).
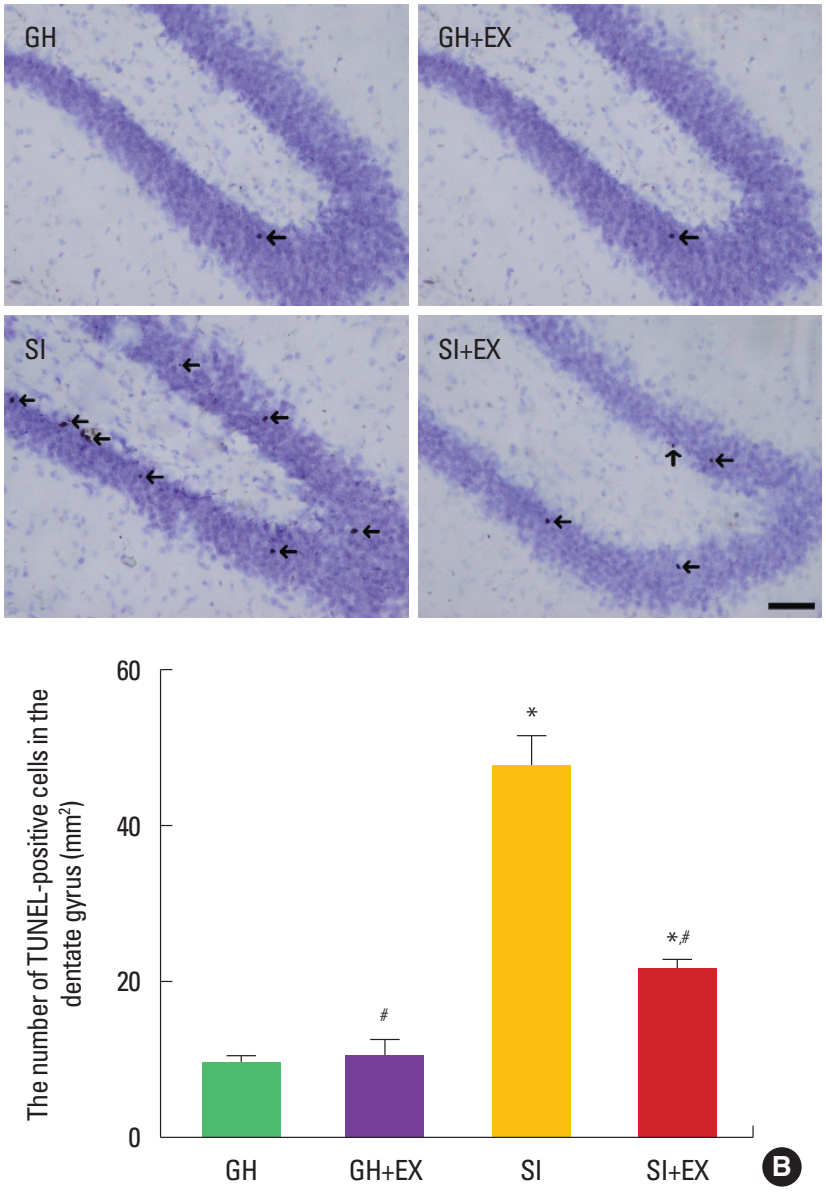

Fig. 2. Effect of swimming exercise on apoptosis in hippocampus. Bax and Bcl-2 (A), TUNEL-positive cells (B). The scale bar represents $50 \mu \mathrm{m}$. GH, group housed; $\mathrm{GH}+\mathrm{EX}$, group housed and exercise; SI, socially isolated; SI+EX, socially isolated and exercise. Data are expressed as the mean \pm standard error of the mean. ${ }^{*} P<0.05$ compared to the GH group. ${ }^{*} P<0.05$ compared to the SI group. 


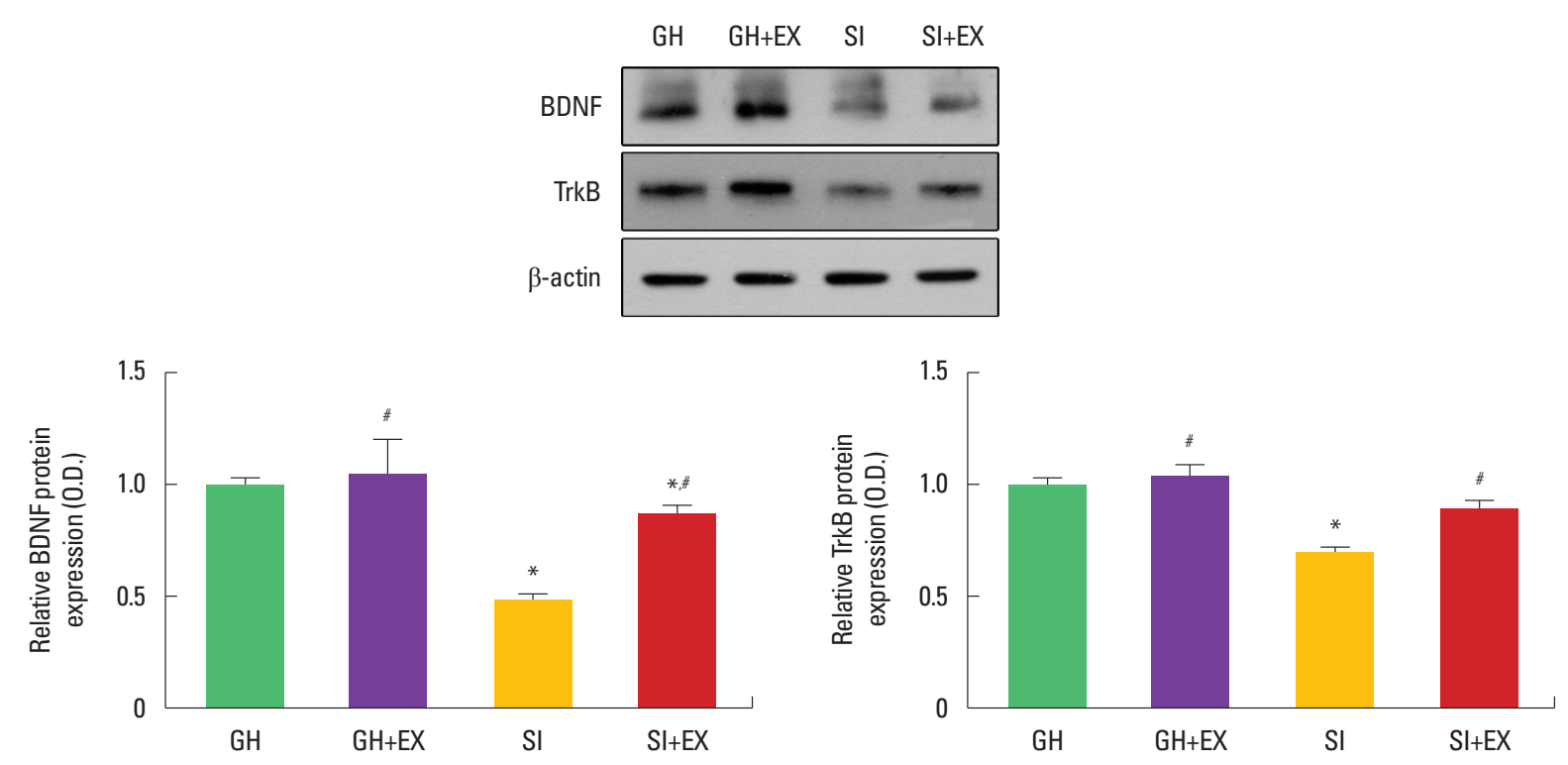

Fig. 3. Effect of exercise on brain-derived neurotrophic factor (BDNF) and tyrosine kinase B (TrkB) in hippocampus. GH, group housed; GH+EX, group housed and exercise; SI, socially isolated; SI+EX, socially isolated and exercise. Data are expressed as the mean \pm standard error of the mean. ${ }^{*} P<0.05$ compared to the GH group. ${ }^{\#} P<0.05$ compared to the Sl group.
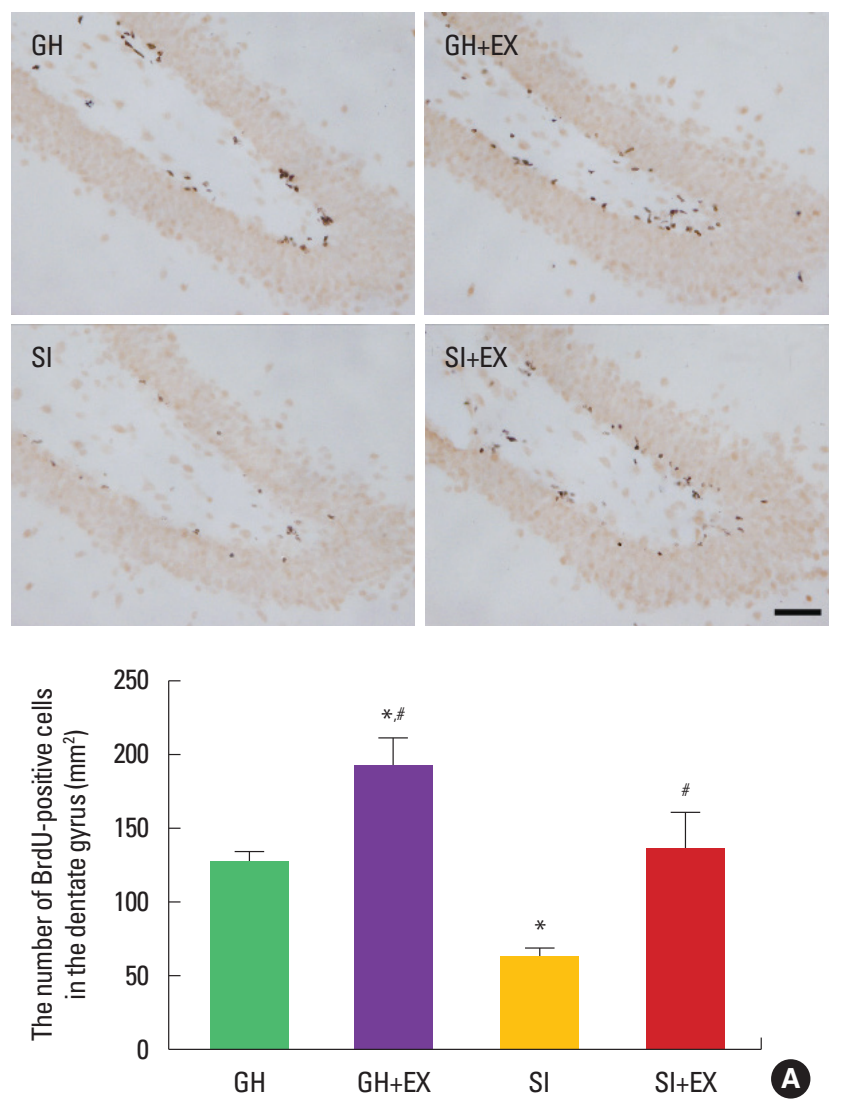
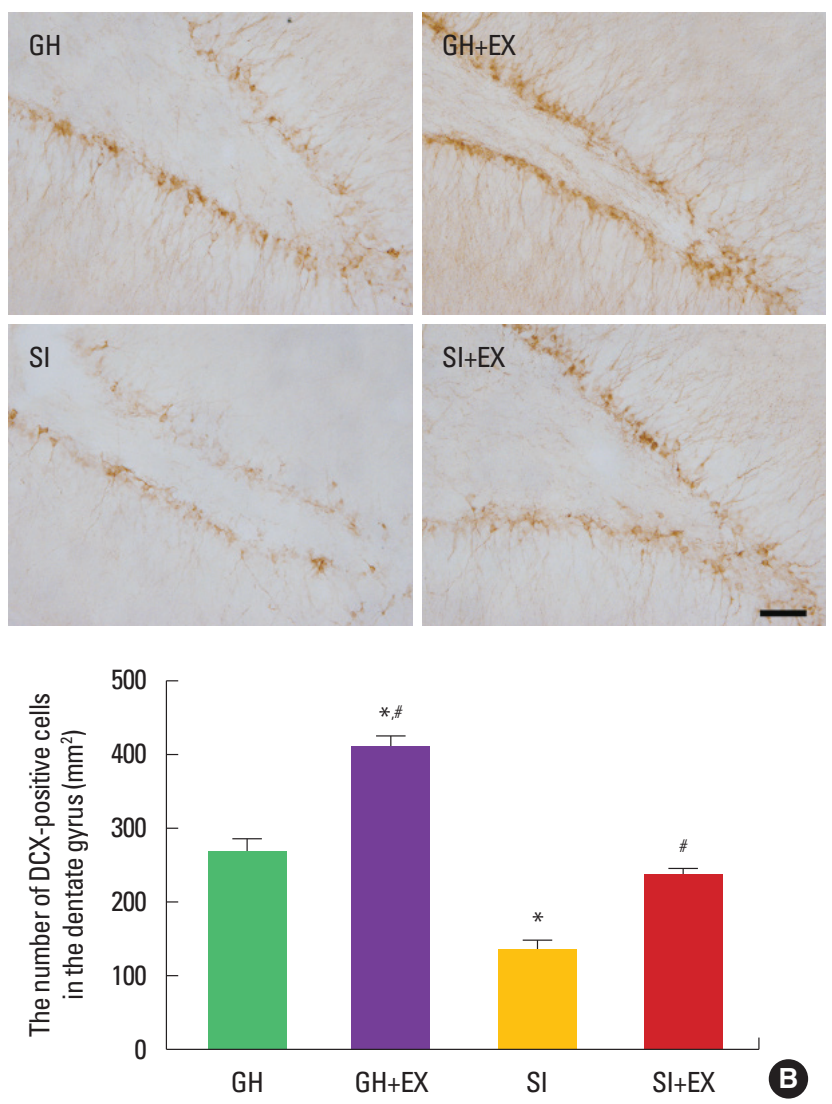

Fig. 4. Effect of exercise on cell proliferation and differentiation in hippocampal dentate gyrus. 5-bromo-2'-deoxyuridine (BrdU)-positive cells (A), doublecortin (DCX)-positive cells (B). The scale bar represents $50 \mu \mathrm{m} \mathrm{GH}$, group housed; GH+EX, group housed and exercise; SI, socially isolated; Sl+EX, socially isolated and exercise. Data are expressed as the mean \pm standard error of the mean. ${ }^{*} P<0.05$ compared to the GH group. ${ }^{\sharp} P<0.05$ compared to the SI group. 

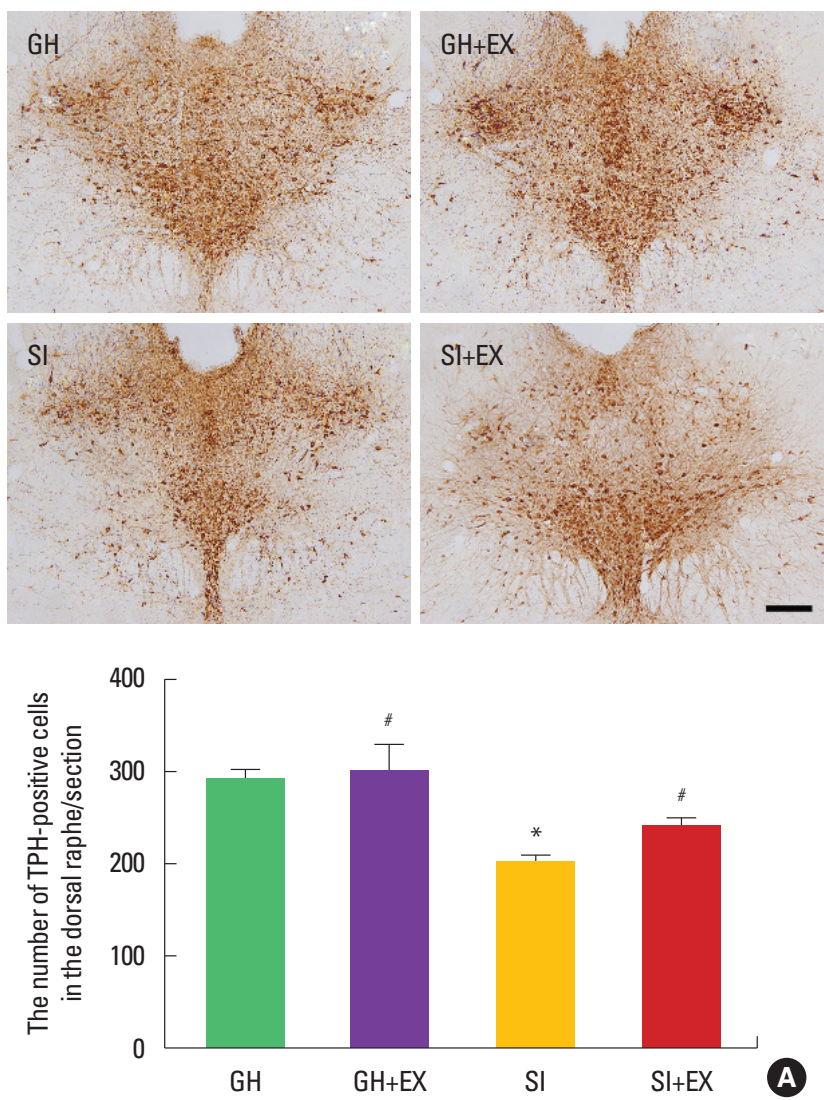
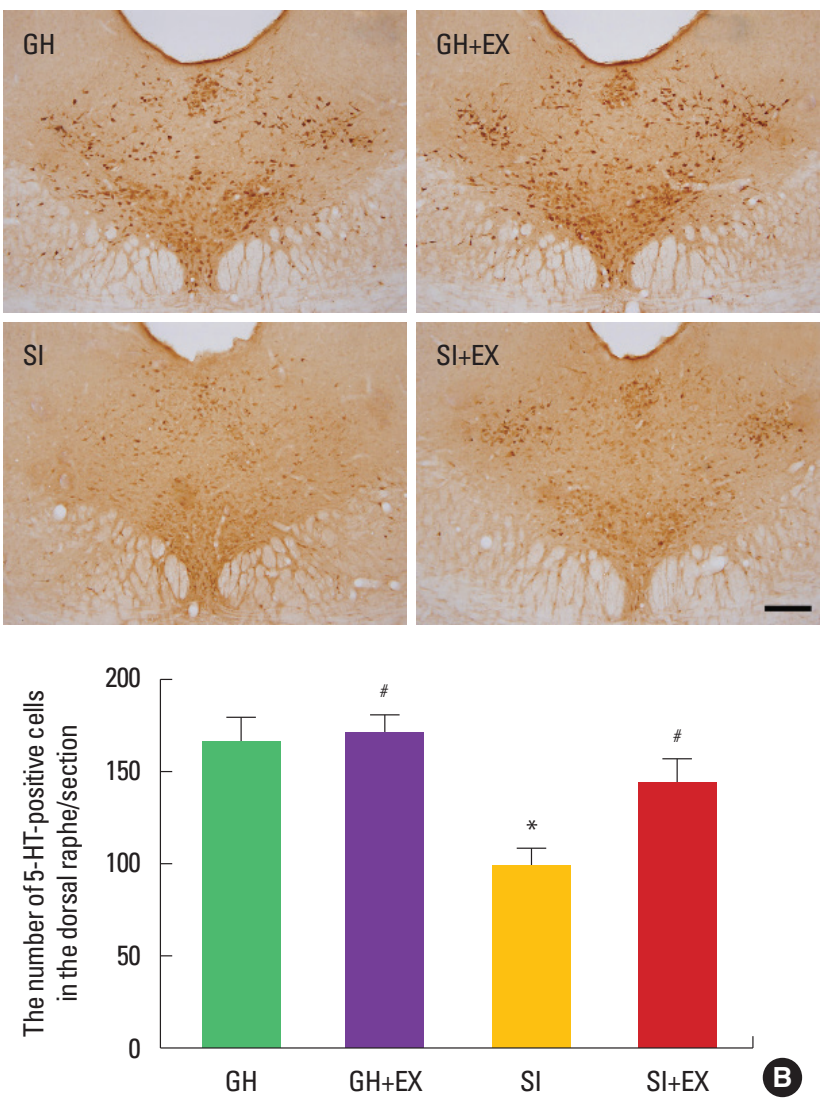

Fig. 5. Effect of swimming exercise on serotonin expression in the dorsal raphe. Tryptophan hydroxylase (TPH)-positive cells (A), 5-hydroxytryptamine (5-HT)-positive cells (B). The scale bar represents $50 \mu \mathrm{m}$. GH+EX, group housed and exercise; SI, socially isolated; SI+EX, socially isolated and exercise. Data are expressed as the mean \pm standard error of the mean. ${ }^{*} P<0.05$ compared to the GH group. ${ }^{*} P<0.05$ compared to the SI group.

\section{DISCUSSION}

Social isolation during adolescence induced serious damage to the brain, leading to reduction in learning ability and spatial memory, causes behavioral change (Larsson et al., 2002). Social isolation decreased memory performance, declines memory capacity, increases negativity, and increased anxious and depressive behaviors (Cacioppo and Hawkley, 2009; Ma et al., 2011). Nitrergic system involved in the behavioral deficits, leading to the development of depression and anxiety (Amiri et al., 2015). In this study, the rats in the social isolation during adolescence stage showed anxiety in elevated plus-maze test, depression in forced swimming test, shortterm memory impairment in step-through avoidance test.

Social isolation and chronic unpredictable mild stress exhibited depressive-like behavior and show a phenotype of mixed apoptosis/autophagy characteristic (Wang et al., 2019). In this study, social isolation during adolescence stage increased Bax expression, decreased $\mathrm{Bcl}-2$ expression, and increased the number of TUNEL- positive cells. These results represent social isolation facilitated apoptosis.

Social isolation significantly reduced BDNF concentration in the hippocampus (Scaccianoce et al., 2006). In this study, social isolation during adolescence stage suppressed BDNF and TrkB expression in the hippocampus.

Social isolation promoted expression of depression-related parameters, which inhibit generation of neurons (Huang et al., 2011). Social isolation stress factors changed neurogenesis in the hippocampus, which could impair spatial memory in adulthood (McCormick et al., 2012). The effect of stress on hippocampal neurogenesis and associated behaviors might be particularly strong during adolescence (Kozareva et al., 2018). In this study, social isolation during adolescence stage suppressed the number of BrdU-positive and DCX-positive cells in the hippocampal dente gyrus. These results represent social isolation inhibited cell proliferation and differentiation.

Social isolation in the adolescent period increased serotonin re- 
lease in the nucleus accumbens (Lukkes et al., 2009). The activation of 5-HT neurons was vulnerable to stress and played an important role in behaviors related to depression and anxiety (Teissier et al., 2015). Social isolation during the adolescent period resulted in decreased excitability of 5-HT neurons in the dorsal raphe (Sargin et al., 2016). In this study, social isolation during adolescence stage inhibited TPH and 5-HT expression in the dorsal raphe.

Exercise after social isolation increased the level of BDNF in the hippocampus and improve depression by increasing serotonergic cells in the raphe nuclei (Hong et al., 2015). Exercise improved mood disorders, including depression and anxiety, and reduces depressive symptoms by increased serotonin expression (Ji et al., 2017; Park et al., 2017). Exercise enhanced hippocampal neurogenesis during adolescence (Kozareva et al., 2018).

In this study, swimming exercise ameliorated anxiety, depression, short-term impairment. Swimming exercise suppressed apoptosis, enhanced neurogenesis, and increased serotonin expression. In our study, swimming exercise ameliorates mood disorder and memory impairment by enhancing neurogenesis and serotonin expression and inhibiting apoptosis in social isolation during adolescence.

\section{CONFLICT OF INTEREST}

No potential conflict of interest relevant to this article was reported.

\section{ACKNOWLEDGMENTS}

This work was supported by the Ministry of Education of the Republic of Korea and the National Research Foundation of Korea (NRF-2016S1A5A2A01027017).

\section{REFERENCES}

Amiri S, Haj-Mirzaian A, Rahimi-Balaei M, Razmi A, Kordjazy N, Shirzadian A, Ejtemaei Mehr S, Sianati H, Dehpour AR. Co-occurrence of anxiety and depressive-like behaviors following adolescent social isolation in male mice; possible role of nitrergic system. Physiol Behav 2015;145:38-44.

Cacioppo JT, Hawkley LC. Perceived social isolation and cognition. Trends Cogn Sci 2009;13:447-454.

Cho JW, Jung SY, Lee SW, Lee SJ, Seo TB, Kim YP, Kim DY. Treadmill exercise ameliorates social isolation-induced depression through neuronal generation in rat pups. J Exerc Rehabil 2017;13:627-633.

Dunphy-Doherty F, O'Mahony SM, Peterson VL, O'Sullivan O, Crispie F,
Cotter PD, Wigmore P, King MV, Cryan JF, Fone KCF. Post-weaning social isolation of rats leads to long-term disruption of the gut microbiota-immune-brain axis. Brain Behav Immun 2018;68:261-273.

Farmer J, Zhao X, van Praag H, Wodtke K, Gage FH, Christie BR. Effects of voluntary exercise on synaptic plasticity and gene expression in the dentate gyrus of adult male Sprague-Dawley rats in vivo. Neuroscience 2004;124:71-79.

Gavrieli Y, Sherman Y, Ben-Sasson SA. Identification of programmed cell death in situ via specific labeling of nuclear DNA fragmentation. J Cell Biol 1992;119:493-501.

Hall J, Thomas KL, Everitt BJ. Rapid and selective induction of BDNF expression in the hippocampus during contextual learning. Nat Neurosci 2000;3:533-535.

Hong YP, Lee HC, Kim HT. Treadmill exercise after social isolation increases the levels of NGF, BDNF, and synapsin I to induce survival of neurons in the hippocampus, and improves depression-like behavior. J Exerc Nutrition Biochem 2015;19:11-18.

Huang HJ, Liang KC, Ke HC, Chang YY, Hsieh-Li HM. Long-term social isolation exacerbates the impairment of spatial working memory in APP/PS1 transgenic mice. Brain Res 2011;1371:150-160.

Ji ES, Lee JM, Kim TW, Kim YM, Kim YS, Kim K. Treadmill exercise ameliorates depressive symptoms through increasing serotonin expression in postpartum depression rats. J Exerc Rehabil 2017;13:130-135.

Kozareva DA, O'Leary OF, Cryan JF, Nolan YM. Deletion of TLX and social isolation impairs exercise-induced neurogenesis in the adolescent hippocampus. Hippocampus 2018;28:3-11.

Larsson F, Winblad B, Mohammed AH. Psychological stress and environmental adaptation in enriched vs. impoverished housed rats. Pharmacol Biochem Behav 2002;73:193-207.

Lee JM, Kim TW, Park SS, Kim CJ, Shin MS, Lee SJ, Kim SH, Baek SS. Wnt signaling pathway is implicated in the alleviating effect of treadmill exercise on maternal separation-induced depression. J Exerc Rehabil 2019;15:200-205.

Lee MH, Kim H, Kim SS, Lee TH, Lim BV, Chang HK, Jang MH, Shin MC, Shin MS, Kim CJ. Treadmill exercise suppresses ischemia-induced increment in apoptosis and cell proliferation in hippocampal dentate gyrus of gerbils. Life Sci 2003;73:2455-2465.

López-Toledano MA, Shelanski ML. Neurogenic effect of beta-amyloid peptide in the development of neural stem cells. J Neurosci 2004;24: 5439-5444.

Lukkes JL, Watt MJ, Lowry CA, Forster GL. Consequences of post-weaning social isolation on anxiety behavior and related neural circuits in rodents. Front Behav Neurosci 2009;3:18.

Ma XC, Jiang D, Jiang WH, Wang F, Jia M, Wu J, Hashimoto K, Dang YH, Gao CG. Social isolation-induced aggression potentiates anxiety and 
depressive-like behavior in male mice subjected to unpredictable chronic mild stress. PLoS One 2011;6:e20955.

McCormick CM, Thomas CM, Sheridan CS, Nixon F, Flynn JA, Mathews IZ. Social instability stress in adolescent male rats alters hippocampal neurogenesis and produces deficits in spatial location memory in adulthood. Hippocampus 2012;22:1300-1312.

McNeal N, Anderson EM, Moenk D, Trahanas D, Matuszewich L, Grippo AJ. Social isolation alters central nervous system monoamine content in prairie voles following acute restraint. Soc Neurosci 2018;13:173-183.

Park HS, Lee JM, Cho HS, Park SS, Kim TW. Physical exercise ameliorates mood disorder-like behavior on high fat diet-induced obesity in mice. Psychiatry Res 2017;250:71-77.

Park SS, Shin MS, Park HS, Kim TW, Kim CJ, Lim BV. Treadmill exercise ameliorates nicotine withdrawal-induced symptoms. J Exerc Rehabil 2019;15:383-391.

Sargin D, Oliver DK, Lambe EK. Chronic social isolation reduces 5-HT neuronal activity via upregulated SK3 calcium-activated potassium channels. Elife 2016;5:e21416.
Scaccianoce S, Del Bianco P, Paolone G, Caprioli D, Modafferi AM, Nencini $\mathrm{P}$, Badiani A. Social isolation selectively reduces hippocampal brain-derived neurotrophic factor without altering plasma corticosterone. Behav Brain Res 2006;168:323-325.

Silva-Gómez AB, Rojas D, Juárez I, Flores G. Decreased dendritic spine density on prefrontal cortical and hippocampal pyramidal neurons in postweaning social isolation rats. Brain Res 2003;983:128-136.

Song SH, Jee YS, Ko IG, Lee SW, Sim YJ, Kim DY, Lee SJ, Cho YS. Treadmill exercise and wheel exercise improve motor function by suppressing apoptotic neuronal cell death in brain inflammation rats. J Exerc Rehabil 2018;14:911-919.

Teissier A, Chemiakine A, Inbar B, Bagchi S, Ray RS, Palmiter RD, Dymecki SM, Moore H, Ansorge MS. Activity of Raphé serotonergic neurons controls emotional behaviors. Cell Rep 2015;13:1965-1976.

Wang M, Bi Y, Zeng S, Liu Y, Shao M, Liu K, Deng Y, Wen G, Sun X, Zeng P, Jing L, Lv Z. Modified Xiaoyao San ameliorates depressive-like behaviors by triggering autophagosome formation to alleviate neuronal apoptosis. Biomed Pharmacother 2019;111:1057-1065. 